\title{
Steigern Schweizer Ärztenetzwerke die Effizienz im Gesundheitswesen?
}

\author{
Ärztenetzwerke werden von vielen Politikern als eine Art Wundermittel gegen das \\ Ausgabenwachstum im Gesundheitswesen angesehen. Selten wird diese Erwartung \\ jedoch auf empirische Resultate abgestützt. Das CSS Institut für Empirische Gesund- \\ heitsökonomie erforscht seit Jahren die Entwicklung der Gesundheitsausgaben in \\ Ärztenetzwerken. Der Beitrag liefert eine Zusammenfassung der Ergebnisse.*
}

\begin{abstract}
Maria Trottmann ${ }^{a}$, Konstantin Beck ${ }^{a, b}$, Ute Kunzeb

a Universität Zürich, Institut für Volkswirtschaftslehre

b CSS Institut für Empirische Gesundheitsökonomie, Luzern
\end{abstract}

* Eine ausführliche Version des Berichtes ist unter www. css-institut.ch zu finden [1].

Korrespondenz:

Maria Trottmann

Universität Zürich

Institut für Volkswirtschaftslehre

Hottingerstrasse 10

CH-8006 Zürich

Tel. 0446344596

maria.trottmann[at]econ.uzh.ch

\section{Methodik}

Werden die Gesundheitsausgaben innerhalb und ausserhalb von Ärztenetzwerken verglichen, ist in erster Linie zu beachten, dass die Versichertenbestände grosse Unterschiede aufweisen können. So kommt es häufig vor, dass Ärztenetzwerke eher die jüngeren und gesünderen Versicherten anziehen. Chronisch erkrankte Personen haben bereits ein Vertrauensverhältnis zu ihrem Arzt oder ihrer Ärztin aufgebaut. Der Wechsel in ein Ärztenetzwerk bedeutet für sie daher eine grössere Umstellung als für gesunde Versicherte. Würde diesem Umstand nicht Rechnung getragen, würden die Einsparungen von Ärztenetzwerken überschätzt. Umgekehrt ist es aber auch möglich, dass ein bestimmtes Ärztenetzwerk einen Patientenstamm mit einem besonders hohen Leistungsbedarf betreut. In diesem Falle würden die Sparleistungen dieses Ärztenetzwerkes unterschätzt. Um Unterschiede im Patientenkollektiv von den «echten» Leistungseinsparungen $\mathrm{zu}$ trennen, braucht es geeignete statistische Methoden.

In diesem Artikel kommen drei unterschiedliche Schätzmethoden zur Anwendung. Die erste wird vom Bundesamt für Gesundheit (BAG) vorgeschrieben, um die Rabatte zu rechtfertigen, die Krankenversicherer für Ärztenetzwerke geben möchten (für weitere Details siehe [2]). Sie basiert auf gewichteten Gruppendurchschnitten. Die zweite Methode wird am treffendsten als «Zwillingsmethode» bezeichnet [3]. Für jeden Versicherten im Ärztenetzwerk wird ein «Zwilling» aus der ordentlichen Obligatorischen Krankenpflegeversicherung (OKP) ausgewählt, welcher die gleichen beobachtbaren Risikomerkmale, aber freie Arztwahl besitzt. So wird das Versichertenkollektiv im Ärztenetzwerk nachsimuliert. Da verschiedene Versicherte existieren, die als «Zwillinge» in Frage kommen, hat die Auswahl etwas Zufälliges. Um den Einfluss des Zufalles zu reduzieren, werden mehrere Zwillingskollektive gezogen und der Durchschnitt all dieser Kollektive verwendet. Die dritte Methode wird als «CART-Methode» bezeichnet. Sie

\section{Est-ce que les réseaux}

\section{de médecins suisses augmentent}

\section{l'efficacité du système de santé?}

\section{Une analyse à moyen terme}

Dans le cadre de cette analyse, nous nous sommes posés la question de savoir si les réseaux de médecins produisent réellement à moyen terme des réductions de coûts significatives. Un aspect important est la distinction entre les économies de coûts causées par la concentration de personnes en meilleure santé dans les réseaux et les économies causées par des traitements plus efficaces. Afin de réaliser cette distinction de manière sérieuse, nous avons appliqué trois méthodes de calcul différentes. Nous avons constaté que les réseaux étudiés sur une période de quatre ans ont produit des économies de coûts considérables, générées réellement par une amélioration de l'efficacité des traitements. Selon la méthode appliquée, le gain d'efficacité se situe en moyenne entre 12 et $20 \%$. L'étude a aussi mis en évidence qu'il existe de grandes différences de résultats entre les réseaux étudiés. Des conclusions globales sur les réductions de coûts produites par tous les réseaux de manière «quasi-automatique» sont remises en question. Un autre résultat de même importance est le fait que les personnes tombées malades ne quittent pas les réseaux de manière plus fréquente que les personnes en bonne santé. Environ quinze ans après l'introduction des réseaux de médecins en Suisse, on constate qu'ils sont bien acceptés par une grande proportion de la population, et qu'ils produisent de réels gains d'efficacité dans les traitements. 
basiert wie die BAG-Methode auf Gruppendurchschnitten. Diesmal wird die Gruppeneinteilung jedoch nicht fix vorgegeben, sondern durch ein software-gestütztes Verfahren optimal vorgenommen. Dieses Verfahren stellt sicher, dass in jeder Gruppe ausreichend Personen für eine stabile Berechnung vorhanden sind.

Bei allen Methoden werden die Versicherten anhand von beobachtbaren Risikomerkmalen gruppiert. Dabei wird davon ausgegangen, dass Versicherte mit den gleichen Risikomerkmalen relativ gut miteinander vergleichbar sind. Die Tabelle 1 gibt eine Übersicht über die verwendeten Risikomerkmale. Während die BAG-Methode sechs verschiedene Risikomerkmale verwendet, sind es bei der Zwillingsoder CART-Methode wesentlich mehr. Als besonders aussagekräftig darf das Merkmal der pharmazeutischen Kostengruppen bezeichnet werden, das chronische Erkrankungen anhand des Medikamentenbezugs identifiziert.

\section{Resultate}

Einsparungen werden traditionellerweise in Prozent angegeben. Bevor auf die Resultate eingegangen wird, soll daran erinnert werden, dass bei Prozentangaben die Wahl der Vergleichsgrösse von zentraler Bedeutung ist. Das Beispiel in Tabelle 2 verdeutlicht dies. Die Durchschnittskosten in der

\begin{tabular}{ll}
$\begin{array}{l}\text { Tabelle } 1 \\
\text { Risikomerkmale zur Gruppierung von Versicherten. }\end{array}$ & $\begin{array}{l}\text { Risikomerkmale } \\
\text { Risikomerkmale } \\
\text { der BAG-Methode } \\
\text { und CART }\end{array}$ \\
Risikozone & Risikozone/Gemeinde \\
\hline Altersgruppe & Altersgruppen \\
\hline Geschlecht & Geschlecht \\
\hline $\begin{array}{l}\text { Gewählte Franchise } \\
\text { Spital- oder Pflegeheim- }\end{array}$ & $\begin{array}{l}\text { Gewählte Franchise } \\
\text { aufenthalt im Vorjahr }\end{array}$ \\
\hline & $\begin{array}{l}\text { Splalaufenthalt im Vorjahr } \\
\text { Vorjahr }\end{array}$ \\
\hline Im Analysejahr verstorben & $\begin{array}{l}\text { Pharmazeutische Kosten- } \\
\text { gruppe (PCG) }\end{array}$ \\
\hline
\end{tabular}

Tabelle 2

Beispiel zur Berechnung der Einsparungen in Prozent.

\begin{tabular}{lllll}
$\begin{array}{l}\varnothing \text { Kosten } \\
\text { OKP }\end{array}$ & $\begin{array}{l}\varnothing \text { Kosten } \\
\text { Netzwerk }\end{array}$ & $\begin{array}{l}\text { Risiko- } \\
\text { bereinigte } \\
\text { Einsparung }\end{array}$ & $\begin{array}{l}\text { Einsparung in } \\
\% \text { der Kosten } \\
\text { OKP }\end{array}$ & $\begin{array}{l}\text { Einsparung } \\
\text { in \% der } \\
\text { Kosten im } \\
\text { Netzwerk }\end{array}$ \\
\hline $3200 \mathrm{CHF}$ & $1300 \mathrm{CHF}$ & $300 \mathrm{CHF}$ & $\begin{array}{l}300 / 3200= \\
9,3 \%\end{array}$ & $\begin{array}{l}300 / 1300= \\
23,1 \%\end{array}$ \\
\hline
\end{tabular}

OKP betragen 3200 Franken, diejenigen im Netzwerk nur 1300 Franken. Die Forscher hätten errechnet, dass 300 Franken «echte», risikobereinigte Einsparungen vorlägen, während der verbleibende Unterschied durch Unterschiede des Patientenstamms zustande

\section{«Es zeigt sich aber auch, dass zwischen den einzelnen Ärztenetzwerken erhebliche Unter- schiede bestehen.»}

kommt. Diese Einsparungen von 300 Franken betragen in Prozent des OKP-Durchschnitts etwa 9\%, in Prozent des Netzwerk-Durchschnitts 23\%.

In dieser Studie werden die Einsparungen jeweils konservativ, d.h. in Prozent des OKP-Durchschnitts angegeben. Es wurden sechs unterschiedliche Netzwerke über vier Jahre (2006-2009) untersucht. Als erstes wichtiges Resultat wurde deutlich, dass zwischen den untersuchten Netzwerken und Jahren erhebliche Unterschiede bestehen. Zweitens hat auch die Wahl der Rechenmethode erheblichen Einfluss auf die Resultate.

Tabelle 3 zeigt das gewichtete Mittel der Einsparungen in den sechs Netzwerken.

Mit der Methode des BAG, die nur eine beschränkte Anzahl an Risikomerkmalen verwendet, werden Einsparungen von durchschnittlich etwa $21 \%$ errechnet, während diese Zahlen bei der Zwillings- oder CART-Methode rund 12 bis $13 \%$ betragen. Es ist zu erwarten, dass die BAG-Methode mit nur sechs Risikomerkmalen eine höhere Einsparung errechnet als Methoden mit sehr vielen Risikomerkmalen. Letztere vermögen Risikovorteile der Netzwerkkollektive besser zu erfassen.

Als Beispiel werden in Abbildung 1 auch die detaillierten Resultate für zwei ausgesuchte Ärztenetzwerke gezeigt (für zusätzliche Resultate sei auf den ausführlichen Bericht verwiesen [1]). Diese Netzwerke haben eine hohe Anzahl an Versicherten, was die Stabilität der statistischen Berechnung erhöht. Bei

\section{Tabelle 3}

Gewichtetes Mittel der Einsparungen in sechs Netzwerken von 2006-2009 für die drei verwendeten Berechnungsmethoden.

\begin{tabular}{llll} 
Methode & BAG & Zwilling & CART \\
$\begin{array}{l}\text { Gewichtetes } \\
\text { Mittel }\end{array}$ & 20,6 & 13,0 & 11,9 \\
\hline Median & 23,2 & 10,7 & 9,1 \\
\hline $\begin{array}{l}\text { Standard } \\
\text { Abweichung }\end{array}$ & 6,7 & 6,7 & 8,6
\end{tabular}




\section{Abbildung 1}

Berechnete Einsparungen in ausgesuchten Netzwerken.
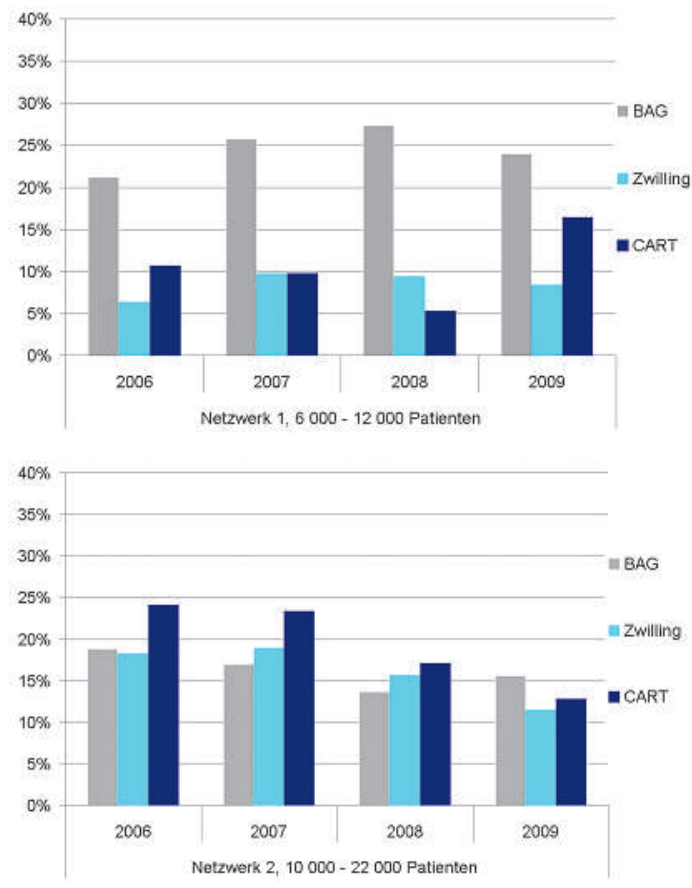

Netzwerk 1 beobachten wir wie erwartet, dass durch die BAG-Methode höhere Einsparungen berechnet wurden als durch die Zwillings- und CART-Methoden. Nach diesen Methoden beträgt die Einsparung zwischen $5 \%$ und $16 \%$. Wie erwähnt sind diese Zahlen in Prozent des OKP Kollektives. Bei dem sehr grossen Netzwerk 2 wurden durch die unterschiedlichen Rechenmethoden ähnliche Resultate erzielt. In den Jahren 2006 und 2007 wurden Einsparungen von 16$24 \%$ gemessen, in den folgenden Jahren sanken die Einsparungen ab auf $12-16 \%$.

Im Durchschnitt waren die Netzwerk-Versicherten in dieser Studie gesünder als die Versicherten in der ordentlichen OKP gemessen am Anteil chronisch kranker Versicherter (in pharmazeutischen Kostengruppen). Es ist interessant zu wissen, ob sich dieser Trend fortsetzt, oder ob Ärztenetzwerke mit zunehmender Bekanntschaft auch mehr Breitenwirkung er-

\section{Abbildung 2}

Durchschnittskosten der Ein- und Austretenden und des Bestandes.

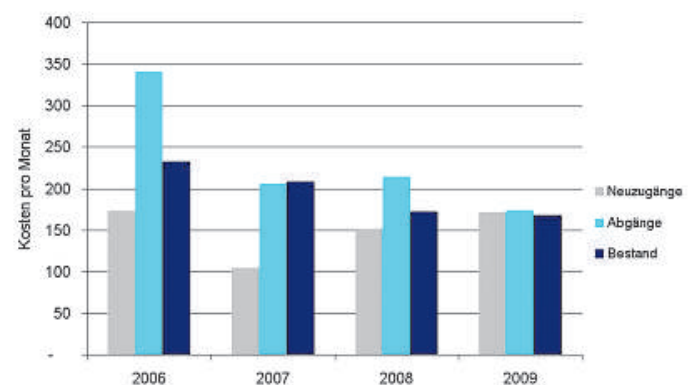

zielen können. Es wäre möglich, dass die Einsparungen der Netzwerke einzig auf den systematischen Ausschluss von chronisch Kranken zurückgeführt werden könnte. Abbildung 2 zeigt die Durchschnittskosten der Eintretenden, Austretenden und des Bestands von 10 Ärztenetzwerken der CSS-Versicherung. In den betrachteten vier Jahren gab es eine klare Dynamik hin zu einer höheren Breitenwirkung der Ärztenetzwerke. Während im Jahr 2006 vor allem günstige Versicherte eintraten und teure Versicherte die Netzwerke verliessen, hatten sich die Kosten der drei Gruppen bis 2009 angeglichen. Der befürchtete Selektionseffekt kann somit mittelfristig nicht nachgewiesen werden. (Dabei wurden auch geographiebedingte Kostenunterschiede kontrolliert.)

\section{Diskussion}

Diese Forschungsarbeit zeigt deutlich, dass die Schweizer Ärztenetzwerke auch auf mittlere Frist erhebliche Einsparungen erzielen und so zur Effizienzsteigerung im Schweizer Gesundheitswesen beitragen. Es zeigt sich aber auch, dass zwischen den einzelnen Ärztenetzwerken erhebliche Unterschiede bestehen. Pauschale Aussagen über die Höhe der Einsparungen, die Ärztenetzwerke «wie automatisch» erzielen, werden so in Frage gestellt. Zukünftige Forschungsartikel sollten sich mit der Frage befassen, was erfolgreiche Ärztenetzwerke auszeichnet bzw. wie die gemessenen Einsparungen erzielt werden. Aus solchen Erkenntnissen könnten auch Handlungsempfehlungen abgeleitet werden.

Ein Nachteil dieser Studie besteht darin, dass die Behandlungsqualität nicht analysiert wurde. Dies ist mit den vorhandenen Daten leider nicht möglich. Als positives Zeichen ist jedoch zu werten, dass diejenigen Versicherten, die 2009 aus Ärztenetzwerken austraten, im Durchschnitt nicht mehr Gesundheitsleistungen beanspruchten als diejenigen Versicherten, die in den Netzwerken verblieben. Da erkrankte Personen besonders gut über die Qualität informiert sind und darauf auch besonderen Wert legen, wäre es ein schlechtes Zeichen, wenn gerade diese Personen die Ärztenetzwerke besonders häufig verlassen würden.

\section{Literatur}

1 Beck K, Kunze U, Buholzer M, Trottmann M. Steigern Schweizer Ärztenetzwerke die Effizienz im Gesundheitswesen? - Eine Analyse auf mittlere Frist. Forschungsbericht des CSS Institut für Empirische Gesundheitsökonomie; 2011 (www.css-institut.ch).

2 Bundesamt für Gesundheit. Kreisschreiben Nr. 5.3 Besondere Versicherungsform mit eingeschränkter Wahl der Leistungserbringer: Nachweis der Kostenunterschiede. Bern; 2011.

3 Beck K, Käser U, Trottmann M, von Rotz S. Effizienzsteigerung dank Managed Care? Evidenz aus der Schweiz. In: datamaster (Beiheft zum Clinicum). 2009; S. 15-21. 